\title{
Electron Cloud Studies for KEKB
}

\author{
G. Rumolo, F. Zimmermann, SL/AP, CERN, Geneva, Switzerland; \\ H. Fukuma, K. Ohmi, KEK, Tsukuba, Japan
}

\begin{abstract}
Electron-cloud build up, incoherent tune spread and electron-induced beam instability are likely to be responsible for the vertical beam-size increase observed at the KEKB Low Energy Ring (LER). We report on recent simulations and analytical estimates, addressing the electroncloud evolution for various magnet configurations, comparing the beam size blow up predicted by three different simulation models (micro-bunches, multi-particle tracking with soft Gaussian approximation, particle-in-cell), and finally discussing analytical expressions for the instability threshold.
\end{abstract}

\section{INTRODUCTION}

Since 1999, a blow up of the vertical beam size is seen at the Low-Energy Ring positron (LER) of the KEK B factory when the current exceeds a certain threshold value, which depends on the filling pattern $[1,2,3]$. At a bunch spacing of $4 \mathrm{rf}$ buckets $(8 \mathrm{~ns})$ the threshold bunch current is about $0.5 \mathrm{~mA}$, equivalent to a bunch population of $N_{b} \approx 3.3 \times$ $10^{10}$. For short bunch trains, the first few bunches after a long gap are of nominal size. The blow up starts around the 7th bunch in a train, as was observed by a gated camera [4]. The evolution of the beam-size blow up coincides with that of the coherent tune shift which equally increases along the train and saturates after about the same number of bunches [5]. During usual luminosity operation, there is a long train of colliding bunches, with a single gap of about $1 \mu \mathrm{s}$. In this condition, there is no significiant difference between the first bunches after the gap and the others, and all bunches appear to blow up more or less equally, which is evidenced by a uniform loss in specific luminosity for all bunches.

The similarity of the blow up measured for short trains with the simulated electron-cloud build up suggested that photoelectrons, and possibly secondary electrons, were the culprits. In order to constrain the electrons to the vicinity of the chamber wall, first 'C-yoke' quadrupole magnets of about $0.3 \mathrm{~T} / \mathrm{m}$ were installed. Motivated by simulations, in the summer of 2000 these magnets were replaced by 50$\mathrm{G}$ solenoids, and further solenoids have been added at the end of the year. Partly as a result of these installations the luminosity of KEKB has increased, which confirms that the electron cloud was indeed limiting the performance.

\section{ELECTRON BUILD UP}

Simulations of the electron cloud build up are performed using the programs and procedures described in Refs. $[6,7$, 8]. A few parameters are listed in Table 1.
Table 1: Selected simulation parameters.

\begin{tabular}{lcc}
\hline chamber radius & $b$ & $47 \mathrm{~mm}$ \\
beam rms-sizes & $\sigma_{x, y, z}$ & $0.4-0.06-4 \mathrm{~mm}$ \\
bunch spacing & $T_{\mathrm{sep}}$ & $8 \mathrm{~ns}$ \\
photon reflectivity & $R$ & $10-100 \%$ \\
photoel. yield & $Y$ & $0.002-0.05 / \mathrm{e}^{+} / \mathrm{m}$ \\
center of photoel. energy & $E_{p e, 0}$ & $7 \mathrm{eV}$ \\
photoel. energy spread & $\sigma_{e}$ & $5 \mathrm{eV}$ \\
max. sec. emission yield & $\delta_{\max }$ & 1.8 \\
energy of max. sec. yield & $\epsilon_{\max }$ & $300 \mathrm{eV}$ \\
synchrotron tune & $Q_{s}$ & 0.015 \\
slippage factor & $\eta$ & $1.8 \times 10^{-4}$ \\
circumference & $C$ & $3000 \mathrm{~m}$ \\
average beta function & $\beta_{y}$ & $15 \mathrm{~m}$ \\
\hline
\end{tabular}

The simulations show that the equilibrium electron line density in a field-free region increases roughly in proportion to the bunch current $[1,9]$. Figures 1-2 display simulated electron volume densities near the center of the beam pipe for a weak periodic quadrupole field and a weak periodic solenoid field. These pictures can be compared with the simulation results for a field-free region presented in Refs. $[1,9,10]$. In all cases, the electron density is strongly fluctuating; the bunches pass near the minima. The equilibrium density for a field-free region is $5-10 \times 10^{11} \mathrm{~m}^{-3}$ $[1,10]$, or roughly ten or fifty times higher than for the quadrupole and solenoid field, respectively. In the 64-ns gap between trains, the central density remains almost constant in case of the quadrupole field. By contrast, it decays with a time constant of about $30 \mathrm{~ns}$ in the field-free region $[1,10]$, where, however, it is rapidly re-established after the first bunch of the second train. Good agreement of the latter behavior with observations was interpreted to mean that the blow up was predominantly due to electrons in many small residual field-free regions [1]. This motivated the (further) installation of tightly packed solenoids.

However, we must note that the simulations do not show any long-term accumulation of electrons, even when realistic solenoid fields are modeled. Therefore, the apparent survival of a large number of electrons over a $1 \mu$ s gap and an additional hysteresis effect with a time constant of $100 \mathrm{~s}$ [2] could not be explained by simulations so far.

Attempts were made to clear the center of the chamber from electrons by special filling patterns. None of these improved the performance, neither in the simulation nor in the experiment. The optimum filling pattern depends on the decay time of the cloud behind a train. In a field-free region the total number of electrons decays over about 50 
ns, and in a weak solenoid field over $100 \mathrm{~ns}$ [9]. If a large component of elastically reflected low-energy electrons is included in the simulation, the decay time for both fieldfree region and solenoid increases to $150-200 \mathrm{~ns}$ [9].

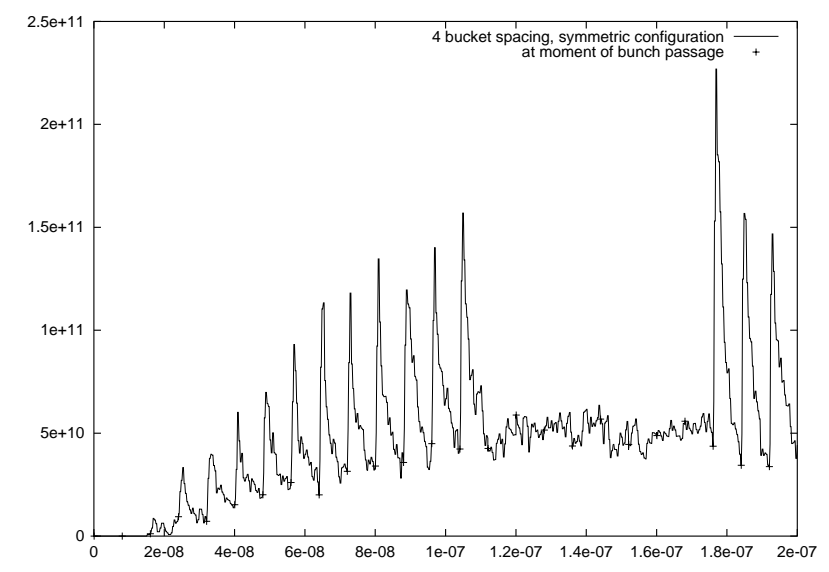

Figure 1: Electron density near beam in units of $\mathrm{m}^{-3}$ as a function of time in seconds, for a perodic quadrupole configuration, with a peak gradient of $0.5 \mathrm{~T} / \mathrm{m}$, minimum 0.1 $\mathrm{T} / \mathrm{m}$ and period $10 \mathrm{~cm}$, during the passage of two bunch trains (4 bucket spacing) with a train-to-train gap of 32 buckets [9].

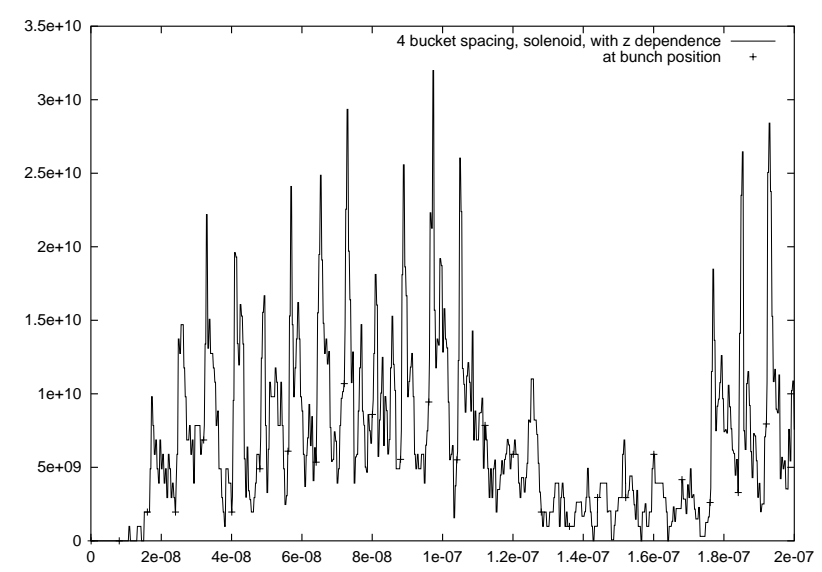

Figure 2: Electron density near beam in units of $\mathrm{m}^{-3}$ for a sinusoidal solenoid field with a peak field of $\pm 50 \mathrm{G}$ and $1-\mathrm{m}$ longitudinal period as a function of time in seconds, during the passage of 20-two bunch trains with 4 bucket bunch-to-bunch spacing and 32-bucket separation between trains [9].

\section{BEAM BLOW UP}

The interaction of the previously established electron cloud with a single bunch is studied in separate simulations $[11,12,13]$. Figures 3 and 4 show a very satisfactory agreement between the soft-Gaussian and the PIC ap- proaches. In particular, the beam vertical size growth is represented for two different values of the electron cloud density. In Fig. 3 the density was assumed to be $\rho_{e}=2.5 \times 10^{11} \mathrm{~m}^{-3}$, and the emittance growth is actually incoherent because this value is below the threshold for the inset of the TMCI. The plots show that in this case there might be a strong dependence of the predicted growth on the number of interaction points between bunch and cloud per turn. It is evident that the emittance, which is predicted to become $15 \%$ higher after $500 \mathrm{~ms}$ if we localize the electron cloud action in one single point along the ring, stays quite constant at its initial value if we split the cloud action over 5 or more different sections.
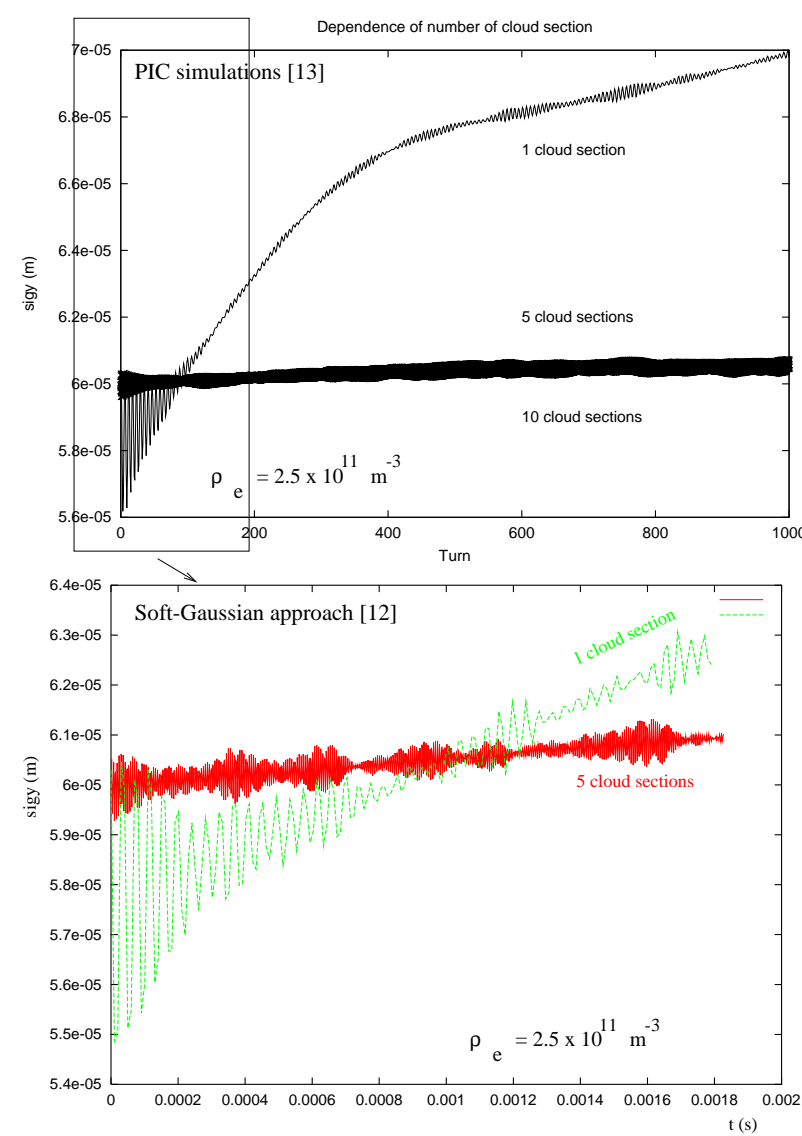

Figure 3: Emittance growth for $\rho_{e}=2.5 \times 10^{11} \mathrm{~m}^{-3}$, with 1 or 5 electron cloud sections along the ring. The simulation with the soft-Gaussian code stops at 200 turns due to the large CPU time required for 5 cloud sections.

In Fig. 4 the emittance growth is shown for the case $\rho_{e}=10^{12} \mathrm{~m}^{-3}$. Again, the profiles look very similar for the PIC and soft-Gaussian approaches. This electron cloud density value is actually above the TMCI threshold, and a coherent vertical motion can be observed. This can be seen in Fig. 5, which depicts the evolution of the centroid vertical position and of the $y z$ correlation normalized to the bunch rms-length over $5 \mathrm{~ms}$ for both cloud densities (see following discussion). 


\section{DISCUSSION}

Without solenoids, a coherent tune shift $\Delta Q_{y}$ of about 0.01 builds up along the bunch train. Estimating, the electron density as $\rho_{e}=2 \gamma \Delta Q_{y} /\left(\beta_{y} C r_{e}\right) \approx 10^{12} \mathrm{~m}^{-3}$, the measured value of $\Delta Q_{y}$ is consistent with the electron density simulated for a field-free region $[3,10]$. Note that the incoherent tune shift can be much larger due to electron accumulation inside the bunch. The measured incoherent tune spread (full width) is of the order 0.03 when solenoids are switched off.

The single-bunch wake field generated by the electron cloud can be approximated by a broad-band resonator [14]. At $\rho_{e}=5 \times 10^{11} \mathrm{~m}^{-3}$, typical resonator parameters for KEKB in the vertical plane are [14] $\omega_{R}=2.2 \times 10^{11} \mathrm{~s}^{-1}$, and $c R_{S} / Q \approx 4 \times 10^{6} \mathrm{~m}^{-2}$. If $\omega_{R} \sigma_{z} / c \gg 1$ the TMCI threshold for a broadband resonator can be estimated as [15] $N_{b, \mathrm{thr}} \approx 5.3 Q_{R} \gamma Q_{s}\left(\omega_{R} \sigma_{z} / c\right)^{2} /\left(c R_{s} / Q\right) / \beta_{y} / r_{e}$, which evaluates to $N_{b, \mathrm{thr}} \approx 3 \times 10^{10}$, assuming $Q_{R} \approx 1$. Note that the threshold scales as $N_{b, \text { thr }} \propto Q_{s} \omega_{R}^{2} \sigma_{z}^{2} / \rho_{e}$. An alternative calculation using the formalism of fast blow up [16] predicts a slightly higher threshold bunch population $N_{b, \mathrm{thr}} \approx 6 \times 10^{10}$; a similar number is obtained by a direct TMCI calculation considering 9 betatron sidebands and ignoring the radial modes (like the estimate above) [14].
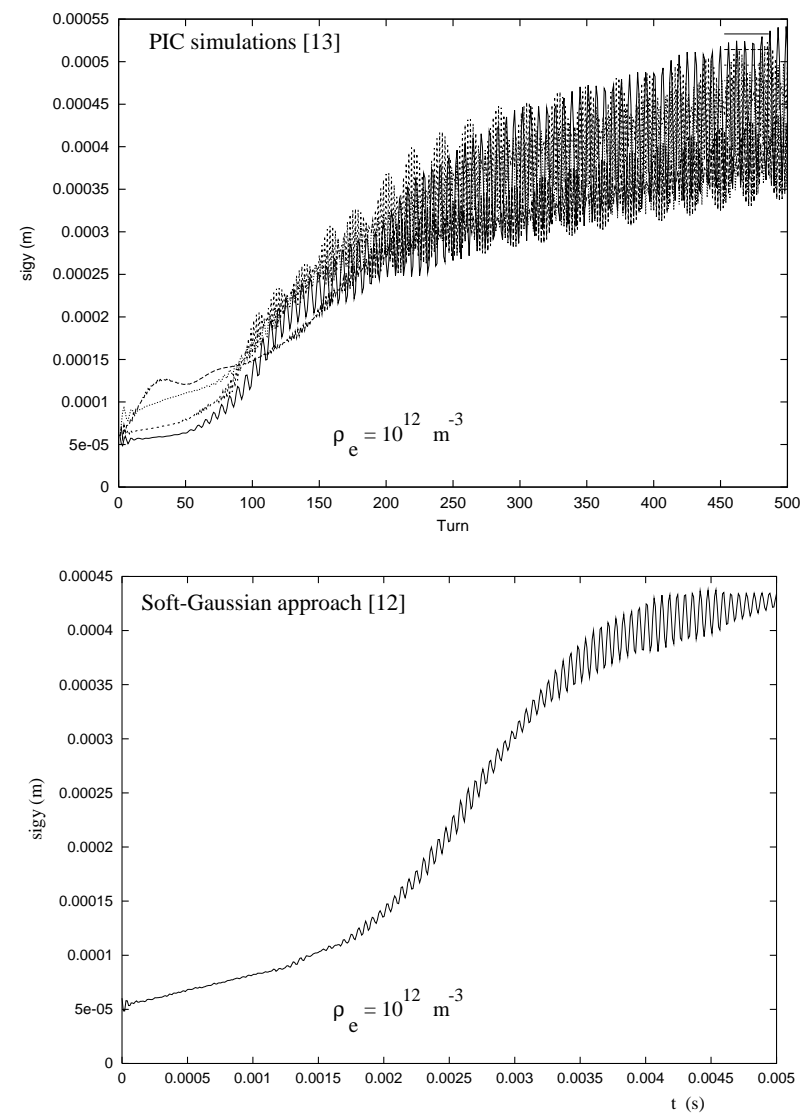

Figure 4: Emittance growth for $\rho_{e}=10^{12} \mathrm{~m}^{-3}$. The different curves in the upper graph represent the evolutions for different betatron tune values.
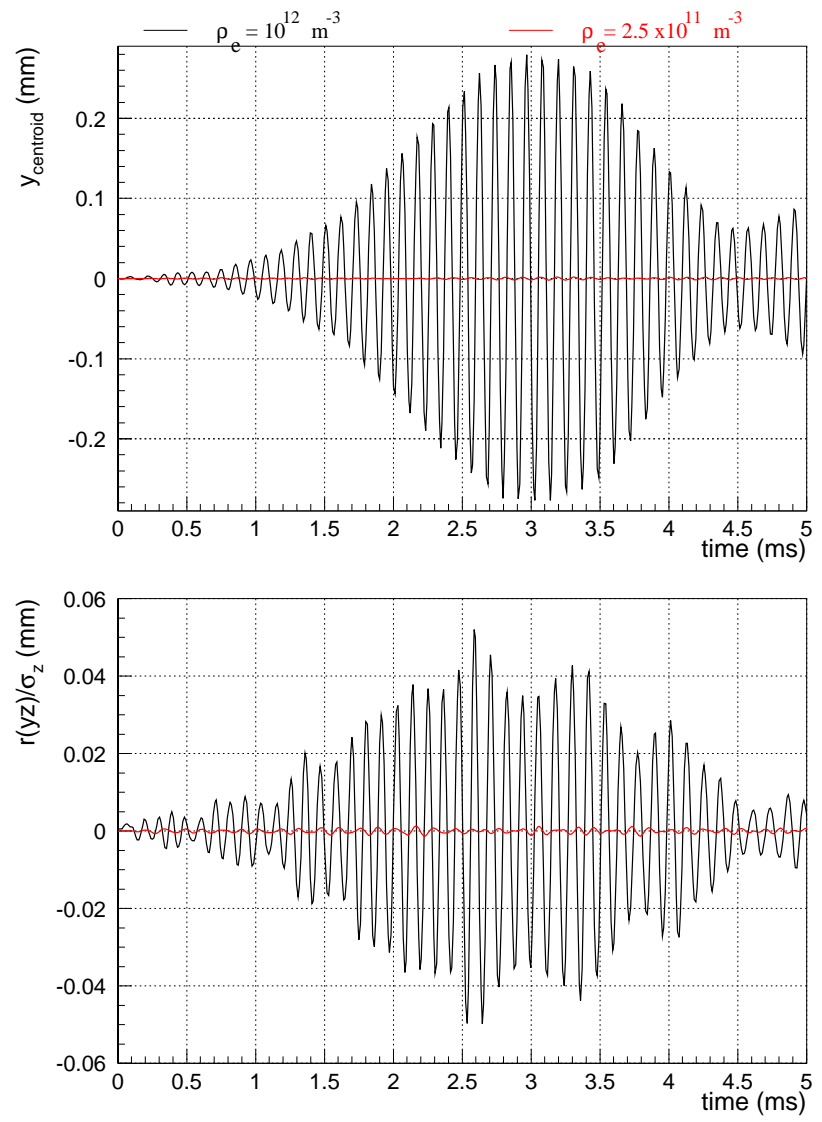

Figure 5: Beam centroid vertical motion and $y z$ correlation versus time for the two cases $\rho_{e}=2.5 \times 10^{11} \mathrm{~m}^{-3}$ and $\rho_{e}=10^{12} \mathrm{~m}^{-3}$. Below threshold, no coherent motion in the $y$-direction is observed.

\section{REFERENCES}

[1] H. Fukuma, et al., EPAC2000 Vienna (2000).

[2] K. Oide, Chamonix XI, CERN-SL-2000-007 DI (2001).

[3] H. Fukuma, et al., HEACC'01 Tsukuba (2001).

[4] J. Flanagan et al., EPAC 2000 Vienna (2000).

[5] T. Ieiri, et al., HEACC01 Tsukuba (2001).

[6] K. Ohmi, Phys. Rev. Lett. 75, 1526 (1995).

[7] G. Rumolo et al., PRST-AB 012801, (2001).

[8] F. Zimmermann, Proc. Chamonix X \& XI, CERN-SL-2000007-DI and CERN-SL-2001-003-DI.

[9] F. Zimmermann, CERN-SL-Note-2000-004 AP; CERN-SL2000-017 (AP); CERN SL-Note-2001-022 AP; F. Zimmermann, H. Fukuma, K. Ohmi, CERN-SL-Note-2000-061 AP.

[10] F. Zimmermann, these proceedings.

[11] K. Ohmi, F. Zimmermann, Phys. Rev. Lett. 85, 3821 (2000).

[12] G. Rumolo, et al., Chamonix XI, CERN-SL-2001-003-DI.

[13] K. Ohmi, these proceedings.

[14] K. Ohmi, et al., HEACC'01 Tsukuba (2001).

[15] B. Zotter, CERN/ISR-TH/82-10 (1982).

[16] R.D. Ruth, J. Wang, IEEE Tr. NS-28, no. 3 (1981); P. Kernel, et al., EPAC 2000, Vienna (2000). 